\title{
New Outlook on the Possible Existence of Superheavy Elements in Nature*
}

\author{
A. Marinov, ${ }^{1}$ 用 S. Gelberg, ${ }^{1}$ D. Kolb, ${ }^{2}$ R. Brandt,${ }^{3}$ and A. Pape ${ }^{4}$ \\ ${ }^{1}$ The Racah Institute of Physics, The Hebrew University, Jerusalem 91904, Israel \\ ${ }^{2}$ Department of Physics, University GH Kassel, 34109 Kassel, Germany \\ ${ }^{3}$ Kernchemie, Philipps University, 35041 Marburg, Germany \\ ${ }^{4}$ IReS-UMR7500, IN2P3-CNRS/ULP, BP28, F-67037 Strasbourg cedex 2, France
}

(Dated: July 23, 2002)

\begin{abstract}
A consistent interpretation is given to some previously unexplained phenomena seen in nature in terms of the recently discovered long-lived high spin super- and hyper-deformed isomeric states. The Po halos seen in mica are interpreted as due to the existence of such isomeric states in corresponding Po or nearby nuclei which eventually decay by $\gamma$ - or $\beta$-decay to the ground states of ${ }^{210} \mathrm{Po},{ }^{214} \mathrm{Po}$ and ${ }^{218} \mathrm{Po}$ nuclei. The low-energy $4.5 \mathrm{MeV} \alpha$-particle group observed in several minerals is interpreted as due to a very enhanced $\alpha$ transition from the third minimum of the potential-energy surface in a superheavy nucleus with atomic number $\mathrm{Z}=108(\mathrm{Hs})$ and atomic mass number around 271 to the corresponding minimum in the daughter.
\end{abstract}

PACS numbers: 23.60.+e, 21.10.Tg, 23.50.+z, 27.90.+b

Keywords: Superheavy elements; Alpha decay; Proton decay; Isomeric states; Superdeformation; Hyperdeformation

\section{INTRODUCTION}

The theoretical predictions in the nineteen sixties

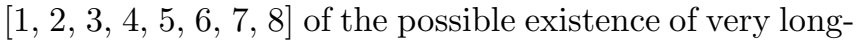
lived superheavy elements around $\mathrm{Z}=114$ and $\mathrm{N}=184$ stirred a lot of excitement among the nuclear scientific community and have initiated the search for the possible existence of superheavy elements in nature. In the present paper we concentrate on two independent well established experimental results which are impossible to understand under the present common knowledge of nuclear physics. These puzzling data are first the observation, in mica minerals, of certain halos which have been attributed to the $\alpha$-decay of the short-lived ${ }^{210} \mathrm{Po},{ }^{214} \mathrm{Po}$ and ${ }^{218}$ Po nuclei [9, 10, 11], and secondly the observation in several minerals of a low energy $\alpha$-particle group with an energy of about $4.5 \mathrm{MeV}[12,13,14,15]$.

Halos in mica, which consist of tiny concentric rings, are known for a long time [16, 17]. For most of them the measured radii of the rings fit with the known ranges of the various $\alpha$-particle groups from ${ }^{238} \mathrm{U}$ or ${ }^{232} \mathrm{Th}$ decay chains. Therefore they have been correctly interpreted, back in 1907 [16, 17, as due to the existence of very small grains of ${ }^{238} \mathrm{U}$ or ${ }^{232} \mathrm{Th}$ in the centers of the corresponding halos, which have been decaying, through their characteristic decay chains, since the time of crystallization of the crust of the earth. However, other types of halos have been discovered back in 1939 [9] and have been thoroughly studied by Gentry [10]. These are the ${ }^{210} \mathrm{Po}$, ${ }^{214} \mathrm{Po}$ and ${ }^{218} \mathrm{Po}$ halos which consist of respective one, two and three concentric rings, with radii equal to the ranges of the $\alpha$-particle groups from the decay chains of

\footnotetext{
* Paper presented at VII Int. School-Seminar on Heavy Ion Physics, May 27 - June 1, 2002. Dubna Russia.

†Electronic address: marinov@vms.huji.ac.i1
}

the corresponding Po isotopes ${ }^{1}$. These Po isotopes belong to the ${ }^{238} \mathrm{U}$ decay chain. However, their half-lives, as well as the half-lives of their $\beta$-decay parents, are short, and since rings belonging to their long-lived precursors are absent, their appearance in nature is puzzling [18].

Another puzzling phenomenon is the low-energy $\alpha$ particle group, around $4.5 \mathrm{MeV}$, which has been seen in molybdenite 12], in thorite [13], in magnetite [14] and in OsIr |15]. The cleanest spectrum, where this group appears without observed residues from $\mathrm{U}$ isotopes decays, was obtained by Cherdyntsev et al. [14]. Based on chemical behavior (having volatile oxides) of the $\alpha$ emitter, it has been suggested that it might be due to a decay of an isotope of Eka-Os, the superheavy element with $\mathrm{Z}=108(\mathrm{Hs}){ }^{2}$ Since it was usually found together with ${ }^{247} \mathrm{Cm}$ and ${ }^{239} \mathrm{Pu}$, it has been suggested [15] that this low-energy $\alpha$-particle group is due to an isotope of element 108 which is a precursor of ${ }^{247} \mathrm{Cm}$ and its descendant ${ }^{239} \mathrm{Pu}$. The half-life of this activity has been estimated to be around $(2.5 \pm 0.5) \times 10^{8}$ y [12].

With the current common knowledge of nuclear physics it seemed impossible to understand these data. The predicted energies of ground state to ground state $\alpha$ transitions for $\beta$ stable Hs nuclei with atomic masses of 274 to 286 are between 9.5 and $6.7 \mathrm{MeV}$ [19, 20, 21], and the predicted half-lives for these energies are between $3 \times 10^{-2}$ $\mathrm{s}$ and $3 \times 10^{2}$ y [22, 23], as compared to half-life of about $5 \times 10^{16}$ y for $4.5 \mathrm{MeV}$. The question is why the nucleus decays with such a low energy $\alpha$-particle when a much higher energy, with a penetrability factor of at least 14

\footnotetext{
${ }^{1}$ Colored pictures of various halos are given in Ref. [11.

2 Actually Cherdyntsev suggested naming element 108 sergenium, based on part of the great silk path in Kazakhstan (name Serika) where the studied mineral molibdenite was found (private communication from Yu. Lobanov.)
} 
orders of magnitude higher, is available.

A second question is how the nucleus can decay with about eight orders of magnitude shorter lifetime than what is predicted 22, 23 from energy versus lifetime relationships for a normal $4.5 \mathrm{MeV} \alpha$-transition (experimentally estimated $2.5 \times 10^{8}$ as compared to predicted $5 \times 10^{16} \mathrm{y}$ ). (A lifetime in the region of $10^{16} \mathrm{y}$ is certainly impossible, since it implies the existence of about $100 \mathrm{mg}$ of material in the studied samples).
In the following paragraphs similar effects seen in the study of various actinide fractions 24 produced via secondary reactions 25], and also in the study of the ${ }^{16} \mathrm{O}+{ }^{197} \mathrm{Au}$ [26, 27] and the ${ }^{28} \mathrm{Si}+{ }^{181} \mathrm{Ta}$ 28] heavy ion reactions, are summarized. Based on the results of all these experiments, a consistent interpretation for the puzzling phenomena seen in nature is suggested. (See also Ref. [29).
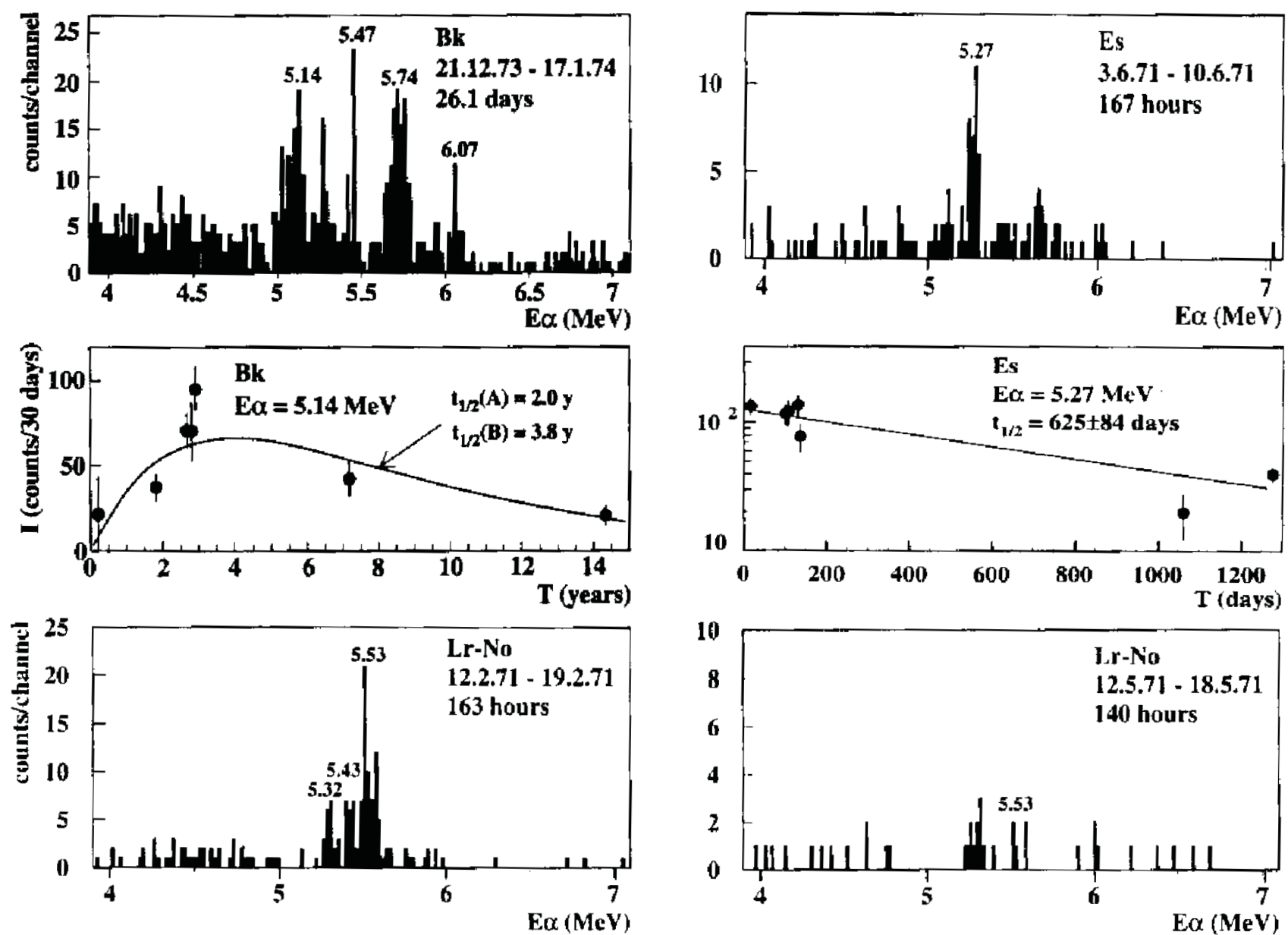

FIG. 1: Left, top: $\alpha$-particle spectrum obtained with the Bk source. Right, top: $\alpha$-particle spectrum obtained with the Es source. Left, center: Decay curve obtained with the $5.14 \mathrm{MeV}$ group seen with the Bk source (left, top above).(See Comment (a) in Table II regarding the growing half-life of 2.0 y [24]). Right, center: Decay curve obtained with the $5.27 \mathrm{MeV}$ group seen with the Es source (right, top above). Left, bottom: $\alpha$-particle spectrum obtained with the Lr-No source. Right, bottom: The same as the previous one but taken about 3 months later. From a comparison of the two spectra a half-life of $26 \pm 7 \mathrm{~d}$ was deduced for the $5.53 \mathrm{MeV}$ group [24].

\section{UNIDENTIFIED $\alpha$-PARTICLE GROUPS IN ACTINIDES}

In a study of actinide fractions from a $\mathrm{W}$ target which had been irradiated with $24-\mathrm{GeV}$ protons, long-lived iso- meric states were found in the neutron-deficient ${ }^{236} \mathrm{Am}$ and ${ }^{236} \mathrm{Bk}$ nuclei with respective half-lives of $0.6 \mathrm{y}$ and $\geq 30 \mathrm{~d}$ [30]. Their character however was not clear, being far from closed shell nuclei, where high spin isomers are known, and living much longer than the known fis- 
sion isomers. In addition, several unidentified $\alpha$-particle groups were found in some actinide sources. Thus, 5.14 $\mathrm{MeV}\left(\mathrm{t}_{1 / 2}=3.8 \pm 1 \mathrm{y}\right), 5.27 \mathrm{MeV}\left(\mathrm{t}_{1 / 2}=625 \pm 84 \mathrm{~d}\right)$ and $5.53 \mathrm{MeV}\left(\mathrm{t}_{1 / 2}=26 \pm 7 \mathrm{~d}\right)$ groups were respectively found in the Bk, Es and Lr-No sources [24, 30] (See Fig. 1 and Table I). Similar to the situation with the $4.5 \mathrm{MeV}$ group seen in nature, also here in the case of the latter unidentified groups, one can not understand their relatively low energies (e.g., $5.53 \mathrm{MeV}$ in Lr-No as compared to typical g.s. to g.s. transitions of around $8 \mathrm{MeV}$ (Column 6 of Table I), which have about 11 orders of magnitude larger penetrability factors (the ratio of Column 4 to Column 7 in Table I)), and their very enhanced character, having $10^{5}-10^{7}$ shorter half-lives than predicted from the systematics of energy versus half-life relationship for normal $\alpha$-decays 22, 23] (See Column 5 in Table I). The deduced evaporation-residue cross sections [24], in the mb region, are also several orders of magnitude larger than expected.

TABLE I: The energies and half-lives of several unidentified $\alpha$-particle groups seen in various actinide sources as compared to theoretical predictions.

\begin{tabular}{|c|c|c|c|c|c|c|}
\hline Source & $(\mathrm{MeV})$ & $\begin{array}{l}\mathrm{t}_{1 / 2}^{\exp } \\
(\mathrm{y}) \\
\end{array}$ & $\begin{array}{l}\mathrm{t}_{1 / 2}^{c a l} 23 \\
(\mathrm{y})\end{array}$ & $\begin{array}{l}\text { Enhance- } \\
\text { ment } \\
\text { factor }^{c}\end{array}$ & $\begin{array}{l}\mathrm{E}_{\alpha}{ }^{a} \\
\text { g.s. } \rightarrow \text { g.s. } \\
(\mathrm{MeV})\end{array}$ & $\mathrm{t}_{1 / 2}^{\text {cal b }}$ \\
\hline $\mathrm{Bk}$ & 5.14 & 3.8 & $1.7 \times 10^{5 d}$ & $4.5 \times 10^{4}$ & $6-7$ & $2.2 \times 10^{9}$ \\
\hline Es & 5.27 & 1.7 & $2.7 \times 10^{6 e}$ & $1.6 \times 10^{6}$ & $7-8$ & $1.9 \times 10^{6}$ \\
\hline No-Lr & 5.53 & 0.07 & $1.1 \times 10^{6 f}$ & $1.5 \times 10^{7}$ & $8-9$ & $2.4 \times 10^{2}$ \\
\hline
\end{tabular}

${ }^{a}$ Typical values from Ref. 33.

${ }^{b}$ Calculated for the lower energy of Column 6 according to formulas given in Ref. [23].

${ }^{c}$ The ratio of Column 4 to Column 3.

${ }^{d}$ Calculated for ${ }^{238} \mathrm{Am}$. See below.

${ }^{e}$ Calculated for ${ }^{247}$ Es. See below.

${ }^{f}$ Calculated for ${ }^{252}$ No. See below.

\section{STUDY OF THE ${ }^{16} \mathrm{O}+{ }^{197} \mathrm{Au}$ REACTION AND LONG-LIVED HIGH SPIN SUPERDEFORMED ISOMERIC STATES}

Possible explanation for the above puzzling data comes from the study of the ${ }^{16} \mathrm{O}+{ }^{197} \mathrm{Au}$ reaction at $\mathrm{E}_{L a b}=80$ $\mathrm{MeV}$ which is around the Coulomb barrier 26, 27], and of the ${ }^{28} \mathrm{Si}+{ }^{181} \mathrm{Ta}$ reaction at $\mathrm{E}_{L a b}=125 \mathrm{MeV}$ [28], about $10 \%$ below the Coulomb barrier. In the first reaction a $5.2 \mathrm{MeV} \alpha$-particle group with a half-life of about $90 \mathrm{~m}$ has been found in ${ }^{210} \mathrm{Fr}$. This group has the same unusual properties as the abnormal $\alpha$-particle groups found in the actinides and produced via secondary reactions, and of the $4.5 \mathrm{MeV}$ found in nature. $5.2 \mathrm{MeV}$ is a low energy as compared to $6.5 \mathrm{MeV}$, the g.s. to g.s. transition from ${ }^{210} \mathrm{Fr}$, and $90 \mathrm{~m}$ half-life is about $4 \times 10^{5}$ enhanced as compared to the prediction [22, 23] for normal $\alpha$-particles of this energy from ${ }^{210} \mathrm{Fr}$. However, the $5.2 \mathrm{MeV}$ group has been found in coincidence with $\gamma$-rays which fit the energies of a superdefermed band transitions. Therefore, the $\alpha$-decay is through a barrier of a superdeformed nucleus, and the large enhancement can quantitatively be understood [26] if one takes into account typical superdeformed radius parameters in the penetrability calculations. The data were consistently interpreted [26] in terms of production of a long-lived high spin isomeric state in the second well of the potential energy surface of ${ }^{210} \mathrm{Fr}$ which decays, by a very enhanced $\alpha$-transition, to a high-spin state in the second well of ${ }^{206} \mathrm{At}$.

The predicted [31, 32] excitation energies of the second minima in ${ }^{210} \mathrm{Fr}$ and nearby nuclei are above the proton separation energies [33]. Therefore, the decay of isomeric states from the second minima, by emitting protons, is in principle possible. In a separate study of the same ${ }^{16} \mathrm{O}+{ }^{197} \mathrm{Au}$ reaction [27], long-lived proton radioactivities with half-lives of about $5.8 \mathrm{~h}$ and $67.3 \mathrm{~h}$ have been discovered. They were interpreted as due to very retarded decays from superdeformed isomeric states in the parent nuclei to normal deformed or to the ground states of the daughters. In particular, the indicated line with $\mathrm{E}_{p}=2.19 \mathrm{MeV}$ [27] may be associated with the predicted 31] $\left(\mathrm{E}_{p}=2.15 \mathrm{MeV}\right)$ second minimum to ground state transition from ${ }^{198} \mathrm{Tl}$, which can be produced by three consecutive superdeformed to superdeformed $\alpha$ transitions from ${ }^{210} \mathrm{Fr}$.

\section{STUDY OF THE ${ }^{28} \mathrm{Si}+{ }^{181}$ Ta REACTION AND LONG-LIVED HIGH SPIN HYPERDEFORMED ISOMERIC STATES}

The ${ }^{28} \mathrm{Si}+{ }^{181} \mathrm{Ta}$ reaction has been studied at $\mathrm{E}_{\text {Lab }}=$ $125 \mathrm{MeV}$, which is about $10 \%$ below the Coulomb barrier, and at $\mathrm{E}_{L a b}=135 \mathrm{MeV}$ [28]. A fusion cross section of about $10 \mathrm{mb}$ is predicted at $125 \mathrm{MeV}$ using a coupledchannel deformation code 34 with deformation parameters $\beta_{2}=0.41$ for ${ }^{28} \mathrm{Si}$ and $\beta_{2}=0.26$ for ${ }^{181} \mathrm{Ta}$ [35] and allowing for $2^{+}$and $3^{-}$excitations in ${ }^{28} \mathrm{Si}$. Only $2 \mu \mathrm{b}$ is predicted when no deformations are included in the calculations. For $135 \mathrm{MeV}$ the corresponding predicted fusion cross sections are $95 \mathrm{mb}$ with deformations and 40 mb without.

Figure 2 (left) shows an $\alpha-\gamma$ two-dimensional coincidence plot obtained at $\mathrm{E}_{L a b}=125 \mathrm{MeV}$. Quite a few coincidence events are seen between a relatively high energy $\alpha$-particle group around $8.6 \mathrm{MeV}$ and various $\gamma$-rays. The half-life of this coincidence group has been measured [28] to be $40 \mathrm{~d} \leq \mathrm{t}_{1 / 2} \leq 2.1 \mathrm{y}$. Figure 2 (right) shows that the $\gamma$-rays which are in coincidence with these highenergy $\alpha$-particles fit nicely with a $\mathrm{J}(\mathrm{J}+1)$ law assuming $\mathrm{E}_{x}=4.42 \times \mathrm{J}(\mathrm{J}+1) \mathrm{keV}$ and $\Delta \mathrm{J}=1$. Based on the observation of a Pt X-ray in coincidence with the $8.6 \mathrm{MeV}$ $\alpha$ 's and on kinematic arguments it was suggested [28] that the $\alpha$-transition is from ${ }^{195} \mathrm{Hg}$ to ${ }^{191} \mathrm{Pt}$. $\left({ }^{195} \mathrm{Hg}\right.$ may be produced via $1 \mathrm{p} 1 \mathrm{n}$ evaporation reaction and 3 consecutive $\mathrm{III}^{\text {min }} \rightarrow \mathrm{III}^{\text {min }} \alpha$-decays. See below). An energy parameter of $4.42 \mathrm{keV}$ is typical to superdeformed band $\gamma$-ray transitions in this region of nuclei. 

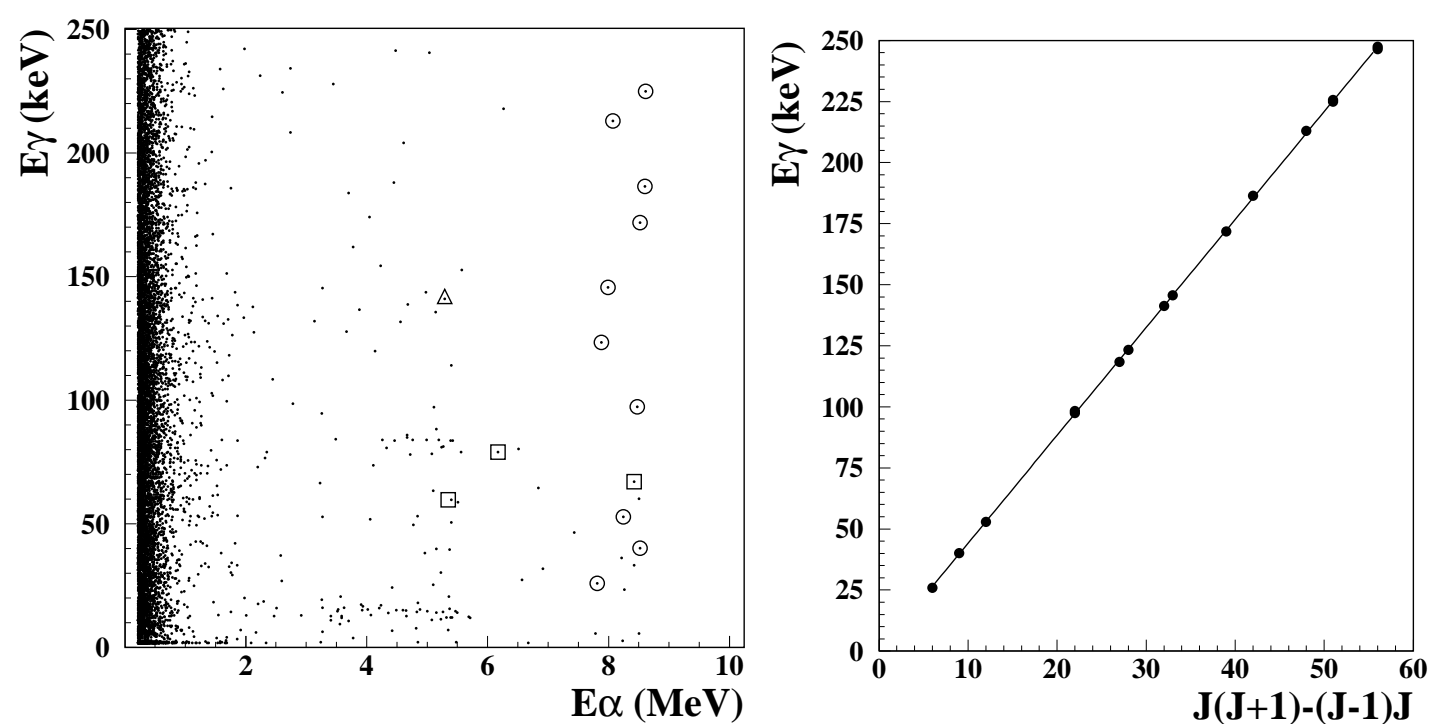

FIG. 2: Left: $\alpha-\gamma$ coincidence plot from one measurement of the ${ }^{28} \mathrm{Si}+{ }^{181} \mathrm{Ta}$ reaction. $\mathrm{E}_{L a b}=125 \mathrm{MeV}$, with $200 \mu \mathrm{g} / \mathrm{cm}^{2}$ $\mathrm{C}$ catcher foil, taken for $76.8 \mathrm{~d}$, starting $77.4 \mathrm{~d}$ after the end of irradiation. The $\gamma$ - ray energies of the encircled events fit with SDB transitions. The squared events fit with known characteristic X - rays and the events in triangles are identified with known $\gamma$ - ray transitions (see Ref. 28]). Right: $\mathrm{E} \gamma$ versus $\mathrm{J}(\mathrm{J}+1)-(\mathrm{J}-1) \mathrm{J}$ for the $\gamma$ - rays seen in coincidence with 7.8 - 8.6 $\mathrm{MeV} \alpha$ - particles. (The encircled events in the left figure plus similar events obtained in a second measurement [28). The slope of the straight line is $4.42 \mathrm{MeV}$.

An $\alpha$-energy of $8.6 \mathrm{MeV}$ is a very high energy for ${ }^{195} \mathrm{Hg}$ which does not decay by emitting $\alpha$-particles and its g.s. to g.s. $Q_{\alpha}$-value is $2.190 \mathrm{MeV}$ [33]. A half-life of $40 \mathrm{~d}$ is about 13 orders of magnitude too long as compared to the systematics of energy versus half-life relationship [23] which predicts $t_{1 / 2} \approx 6 \times 10^{-8} \mathrm{~s}$. Since the $\alpha$ particles are in coincidence with a superdeformed band $\gamma$-ray transitions, the $\alpha$-decay is to the superdeformed well of the daughter nucleus. However, it could not be a $\mathrm{II}^{\text {min }} \rightarrow \mathrm{II}^{\text {min }}$ transition, since such a transition is very enhanced as opposed to the large retardation measured in the experiment. A consistent interpretation, both from the point of view of the high energy of the $\alpha$-particles and their very long lifetime, is that the decay is from a longlived high spin $(\mathrm{J} \approx 39 / 2)$ isomeric state in the $\mathrm{III}^{\mathrm{min}}$, the hyperdeformed minimum [36, 37, 38, 39] of ${ }^{195} \mathrm{Hg}$, which decays by strongly retarded transition to the $\mathrm{II}^{\mathrm{min}}$ of the potential in ${ }^{191} \mathrm{Pt}$ 28]. As seen in Fig. 3 the predicted $Q_{\alpha}$-value for such a transition is about $8.7 \mathrm{MeV}$, taking into account an extrapolated value from Ref. [37] for the excitation energy of the III ${ }^{\text {min }}$ in ${ }^{195} \mathrm{Hg}$ and the predictions of Refs. 31, 32] for the excitation energy of the $\mathrm{II}^{\mathrm{min}}$ in ${ }^{191} \mathrm{Pt}$. This value fits rather nicely with the measured $Q_{\alpha}$-value of about $8.8 \mathrm{MeV}$. (The excitation energy of the state in the third minimum of ${ }^{195} \mathrm{Hg}$ was assumed to be around the rotational $39 / 2$ state with estimated energy of $\mathrm{E}_{x}=2.2 \times \mathrm{J}(\mathrm{J}+1) \mathrm{keV}[39)$.

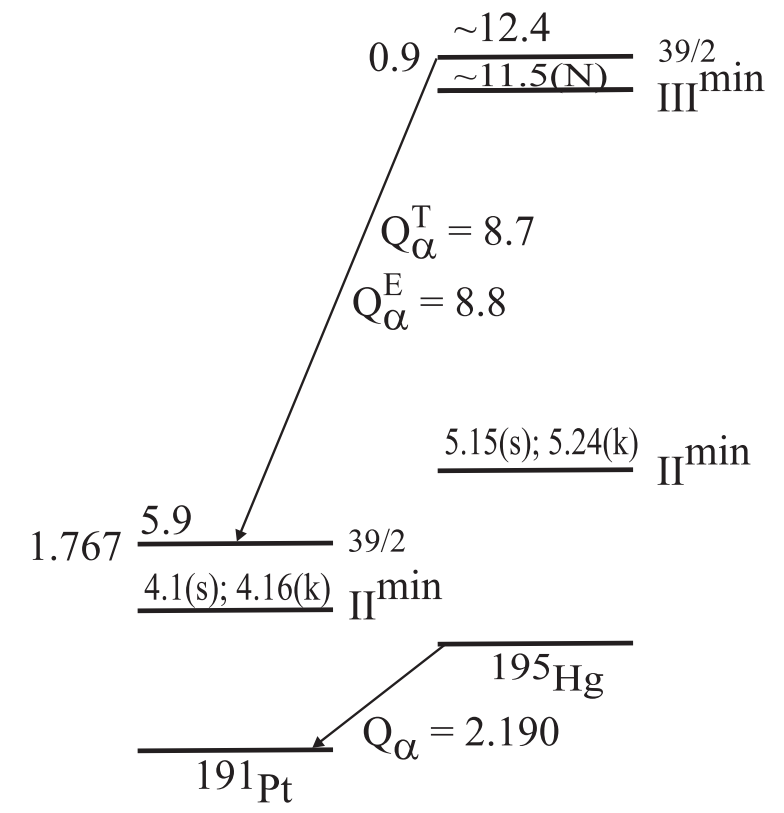

FIG. 3: Proposed decay scheme deduced from the observation of the $8.6 \mathrm{MeV} \alpha$-particles seen in coincidence with a superdeformed band transitions. s - Satula et al. (Ref. 31]); k - Krieger et al. (Ref. 32]); N - Nazarewicz (Ref. [37]).

\section{SUPER- AND HYPER-DEFORMED ISOMERIC STATES IN THE ACTINIDE REGION}

Based on the discovery of the long-lived super- and hyper-deformed isomeric states (Secs. III and IV), a consistent interpretation has been given [24] to the 
unidentified $\alpha$-particle groups seen in the actinide sources and described in Sec. II above. $\mathrm{II}^{\text {min }} \rightarrow \mathrm{II}^{\text {min }}$ and $\mathrm{III}^{\text {min }} \rightarrow \mathrm{III}^{\text {min }} \alpha$-particle transition energies have been deduced from the predicted 36] excitation energies. Table II shows that the low energy 5.14, 5.27 and 5.53 $\mathrm{MeV}$ groups seen in the Bk, Es and No-Lr sources, can consistently be interpreted as due to the low-energy $\mathrm{II}^{\text {min }} \rightarrow \mathrm{II}^{\text {min }}$ transition from ${ }^{238} \mathrm{Am}$ and $\mathrm{III}^{\text {min }} \rightarrow \mathrm{III}^{\text {min }}$ transitions from ${ }^{247}$ Es and ${ }^{252} \mathrm{No}$, respectively. Their energies are considerably lower than the corresponding g.s. to g.s. transitions (Column 6 in Table II).

TABLE II: A comparision between the experimental $\alpha$ particle energies and values deduced from the predictions of Ref. [36] for some superdeformed (SD) to superdeformed and hyperdeformed (HD) to hyperdeformed isomeric transitions. The last column shows the corresponding experimental g.s. to g.s. transitions 33.

\begin{tabular}{llllll}
\hline $\begin{array}{l}\text { Source } \\
\mathrm{E}_{\alpha}^{\exp } \\
(\mathrm{MeV})\end{array}$ & Isotope Transition & $\begin{array}{l}\mathrm{E}_{\alpha}^{\text {cal }} \sqrt{36} \\
(\mathrm{MeV})\end{array}$ & $\begin{array}{l}\mathrm{E}_{\alpha}^{\text {g.s. } \rightarrow \text { g.s. }} \\
(\mathrm{MeV})\end{array}$ \\
\hline $\mathrm{Bk}$ & 5.14 & ${ }^{238} \mathrm{Am}^{a}$ & $\mathrm{SD} \rightarrow \mathrm{SD}$ & 5.13 & 5.94 \\
$\mathrm{Es}$ & 5.27 & ${ }^{247} \mathrm{Es}$ & $\mathrm{HD} \rightarrow \mathrm{HD}$ & 5.27 & 7.32 \\
$\mathrm{No}-\mathrm{Lr}$ & 5.53 & ${ }^{252} \mathrm{No}$ & $\mathrm{HD} \rightarrow \mathrm{HD}$ & $\approx 5.6^{b}$ & 8.42 \\
\hline
\end{tabular}

${ }^{a}$ Since the intensity of the $5.14 \mathrm{MeV}$ group (Fig. 1) grew at the beginning with time, it was assumed here 24 similar to the situation with the isomeric states in ${ }^{236} \mathrm{Bk}$ and ${ }^{236} \mathrm{Am}\left[30\right.$, that ${ }^{238} \mathrm{Bk}$ decayed by EC or $\beta^{+}$transitions to ${ }^{238} \mathrm{Am}$.

${ }^{b}$ Extrapolated value. See Ref. 24 .

Table III shows that the very enhanced measured halflives of the low-energy $\alpha$-particle groups seen in the various actinide sources are consistent with calculated values [24], taking into account in the penetrability calculations the deformation parameters of the superdeformed and hyperdeformed isomeric states.

TABLE III: Experimental and predicted half-lives for superdeformed (SD) to superdeformed and hyperdeformed (HD) to hyperdeformed transitions [24].

\begin{tabular}{llllllll}
\hline $\begin{array}{l}\text { Mother } \mathrm{E}_{\alpha} \\
\text { Isotope }(\mathrm{MeV})\end{array}$ & Transition $\beta_{2}{ }^{a}$ & $\beta_{3}{ }^{a}$ & $\beta_{4}{ }^{a}$ & $\begin{array}{l}\mathrm{t}_{1 / 2}^{\text {cal }} b^{b} \\
(\mathrm{y})\end{array}$ & $\begin{array}{l}\mathrm{t}_{1 / 2}^{e x p} \\
(\mathrm{y})\end{array}$ \\
\hline${ }^{238} \mathrm{Am}$ & 5.14 & $\mathrm{SD} \rightarrow \mathrm{SD}$ & 0.71 & 0.0 & 0.09 & 10.9 & $3.8 \pm 1.0$ \\
${ }^{247} \mathrm{Es}$ & 5.27 & $\mathrm{HD} \rightarrow \mathrm{HD}$ & 1.05 & 0.19 & 0.0 & 1.15 & $1.7 \pm 0.2$ \\
${ }^{252} \mathrm{No}$ & 5.53 & $\mathrm{HD} \rightarrow \mathrm{HD}$ & 1.2 & 0.19 & 0.0 & 0.22 & $0.07 \pm 0.02$ \\
\hline
\end{tabular}

${ }^{a} \beta_{2}$ and $\beta_{4}$ values were deduced from the $\epsilon_{2}$ and $\epsilon_{4}$ values given in Ref. [36] using Fig. 2 of Ref. [40]. The value of $\beta_{3}$ was taken equal to $\epsilon_{3}$.

${ }^{b}$ Calculated according to formulas given in Ref. 24]. Calculated half-lives for other deformation parameters are given in Ref. 24].

The potential energies as function of quadrupole deformations, taking from Ref. [36], are shown in Fig. 4 for the ${ }^{238} \mathrm{Am},{ }^{238} \mathrm{Cm},{ }^{247} \mathrm{Es}$, and ${ }^{248} \mathrm{Fm}$ nuclei. It is seen that in ${ }^{238} \mathrm{Am}$ the inner and the outer barriers of the second minimum are quite large, while in ${ }^{247} \mathrm{Es}$ and ${ }^{248} \mathrm{Fm}$ the outer barriers of the second minima are small, and the inner barriers of the third minima are large. In fact, the third minima in ${ }^{247} \mathrm{Es}$ and ${ }^{248} \mathrm{Fm}$ are predicted to be the ground states of these nuclei, being 0.61 and $1.76 \mathrm{MeV}$ below the normal, slightly deformed, ground states. Unfortunately there are no predictions in these cases for the potential at even larger deformations, beyond the third minimum. (In the case of ${ }^{232} \mathrm{Th}$ 38 the outer barrier in the third minimum is quite high).
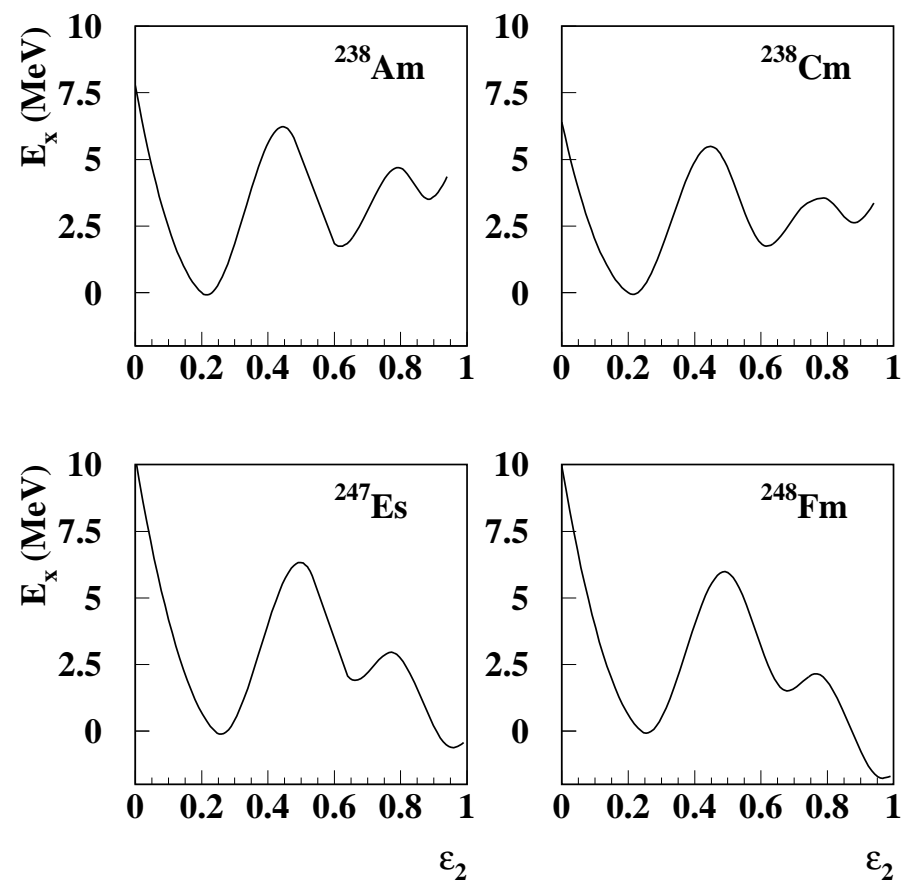

FIG. 4: Potential energies as function of quadrupole deformations for 4 nuclei according to Ref. 36.

In Ref. 24] detailed estimates for the various production cross sections of the actinide nuclei, as well as of the superheavy element with $\mathrm{Z}=112$ and $\mathrm{N} \simeq 160$, are given. It is argued that the relatively large fusion cross sections, in the mb region, are due to two effects. First, the compound nucleus is produced in an isomeric state in the second or third minimum of the potential, rather than in the normal ground state. As shown in Fig. 5 much less overlapping and penetration are needed under these conditions, and therefore the compound nucleus formation probability increases drastically. Secondly, in the secondary reaction experiment the projectile is a fragment which has been produced within $2 \times 10^{-14} \mathrm{~s}$ before interacting with another $\mathrm{W}$ nucleus in the target. During this short time it is at high excitation energy and quite deformed. Figure 6 gives the results of couple-channel calculations 34 for the fusion cross section as function of bombarding energy for the ${ }^{70} \mathrm{Zn}+{ }^{186} \mathrm{~W}$ reaction, taking into account the known deformation of ${ }^{186} \mathrm{~W}$ and various deformations of the projectile. Figure $6 \mathrm{~d}$ shows the results when the projectile has a deformation which is typical for the second minimum of the potential. It is 


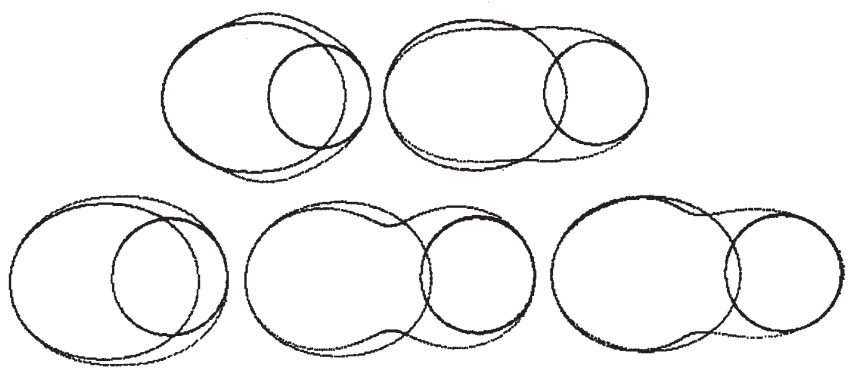

FIG. 5: Calculated shapes of two compound nuclei at various configurations together with the shapes of the corresponding projectile and target nuclei. Top, left: $\mathrm{A}_{C . N}=239$ in the normal ground state; $\beta_{2}=0.2 ; \beta_{4}=0.08$ 36. Top, right: $A_{C . N .}=239$ in the second minimum; $\beta_{2}=0.77 ; \beta_{4}=0.1$ [36]. In both figures: $\mathrm{A}_{\text {heavy }}=186 ; \beta_{2}=0.22$ [35]. $\mathrm{A}_{\text {light }}$ $=53 ; \beta_{2}, \beta_{3}, \beta_{4}=0.0$. Bottom, left: $\mathrm{A}_{C . N}=253$ in the normal ground state; $\beta_{2}=0.28 ; \beta_{4}=0.01$ 36. Bottom, center: $\mathrm{A}_{C . N}=253$ in the third minimum; $\beta_{2}=1.2 ; \beta_{4}=$ 0.0 [36]. Bottom, right: $\mathrm{A}_{C . N}=253$ with parameters of the third minimum of ${ }^{232} \mathrm{Th} ; \beta_{2}=0.85 ; \beta_{3}=0.35 ; \beta_{4}=0.18$ [38]. In the three figures at the bottom: $\mathrm{A}_{\text {heavy }}=186 ; \beta_{2}=$ 0.22 [35]. $\mathrm{A}_{\text {light }}=67 ; \beta_{2}, \beta_{3}, \beta_{4}=0.0$.

seen that, due to the reduced Coulomb repulsion between the two nuclei for the tip to tip configuration, the cross section reduces very slowly with decreasing bombarding energy.

An idea about the relative importance of the above two effects can be deduced from the following arguments: The difference from a typical cross section of about $1 \mathrm{pb}$ 41] obtained in the ${ }^{70} \mathrm{Zn}+{ }^{208} \mathrm{~Pb}$ reaction producing the nucleus ${ }^{277} 112$ in its ground state, to a cross section of about $20 \mathrm{nb}$ producing ${ }^{271(2)} 112$ in an isomeric state via the ${ }^{88} \mathrm{Sr}+{ }^{184} \mathrm{~W}$ reaction 42 , is due to the first effect. The additional difference from $20 \mathrm{nb}$ to about $3.8 \mathrm{mb}$ [24] of producing element 112 in an isomeric state via secondary reactions, is due to the second effect.

\section{SUMMARY OF THE PROPERTIES OF THE SUPER- AND HYPER-DEFORMED ISOMERIC STATES}

Figure 7 summarizes the results obtained about the super- and hyper-deformed isomeric states. The nucleus may have a long lifetime in its ground state, but also in long-lived isomeric states in the second and third minima of the potential-energy surfaces. Long-lived superdeformed isomeric states may decay by very enhanced $\alpha$ particles to superdeformed states in the daughter nuclei, or by strongly retarded $\alpha$-particles to the normal deformed states in the corresponding nuclei. It also may decay by very retarded proton radioactivity. Similarly, hyperdeformed isomeric states may decay by strongly enhanced $\alpha$-particle decay to the hyperdeformed potential well in the daughter, or by very retarded $\alpha$-decay to the

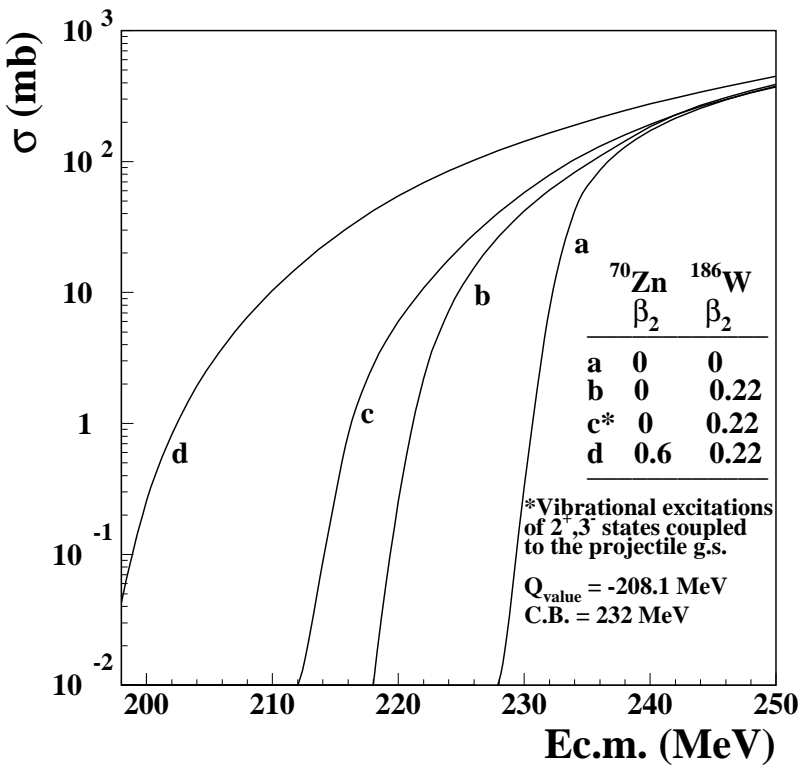

FIG. 6: Calculated fusion cross sections using the Code CCDEF 34 for the ${ }^{70} \mathrm{Zn}+{ }^{186} \mathrm{~W}$ reaction assuming various quadrupole deformations of the projectile and target nuclei (see text, Sec. V).

superdeformed minimum in the same nucleus. All these extremely unusual decay properties have been discovered experimentally as summarized in Fig. 7 .

It should be mentioned that the half-lives of the newly discovered isomeric states are longer than those of their corresponding ground states. Such a comparison for the isomeric states in the actinide region is presented in Table IV.

It should be mentioned that back in 1969 [4] a new type of fission isomeric state has been predicted for nuclei with $\mathrm{N} \approx 144-150$, A specialization energy in excess of 4 $\mathrm{MeV}$ for the second barrier was predicted for a [505] $\frac{11}{2}^{-}$ state, which is associated with a factor of about $10^{15}$ increase in the half-life of a normal fission shape isomer.

TABLE IV: Half-lives of some isomeric states and their ratios to the half-lives of their corresponding normal-deformed ground states.

\begin{tabular}{lllc}
\hline Isotope & $\mathrm{t}_{1 / 2}^{\text {g.s. }}$ & $\mathrm{t}_{1 / 2}^{\text {i.s. }}$ & $\mathrm{t}_{1 / 2}^{\text {i.s. }} / \mathrm{t}_{1 / 2}^{\text {g.s. }}$ \\
\hline${ }^{236} \mathrm{Bk}$ & $42.4 \mathrm{~s}^{a}$ & $\geq 30 \mathrm{~d}^{b} \geq 6.1 \times 10^{4}$ \\
${ }^{236} \mathrm{Am}$ & $3.6 \mathrm{~m}^{c}$ & $219 \mathrm{~d}^{b}$ & $8.8 \times 10^{4}$ \\
${ }^{238} \mathrm{Am}^{d} 98 \mathrm{~m}^{e}$ & $3.8 \mathrm{y}$ & $2.0 \times 10^{4}$ \\
${ }^{247} \mathrm{Es}^{f}$ & $4.55 \mathrm{~m}^{e}$ & $625 \mathrm{~d}$ & $2.0 \times 10^{5}$ \\
${ }^{252} \mathrm{No}^{g}$ & $2.3 \mathrm{~s}^{e}$ & $26 \mathrm{~d}$ & $9.8 \times 10^{5}$ \\
\hline
\end{tabular}

${ }^{a}$ Predicted by P. Möller et al. 19.

${ }^{b}$ Ref. B30.

${ }^{c}$ Y. Nagame et al., this proceedings.

${ }^{d}$ Assuming that the $5.14 \mathrm{MeV}$ is from ${ }^{238} \mathrm{Am}$ (see Tables 1 and 3). ${ }^{e}$ Ref. [43].

${ }^{f}$ Assuming that the $5.27 \mathrm{MeV}$ is from ${ }^{247} \mathrm{Es}$ (see Tables 1 and 3 ).

${ }^{g}$ Assuming that the $5.53 \mathrm{MeV}$ is from ${ }^{252}$ No (see text). 


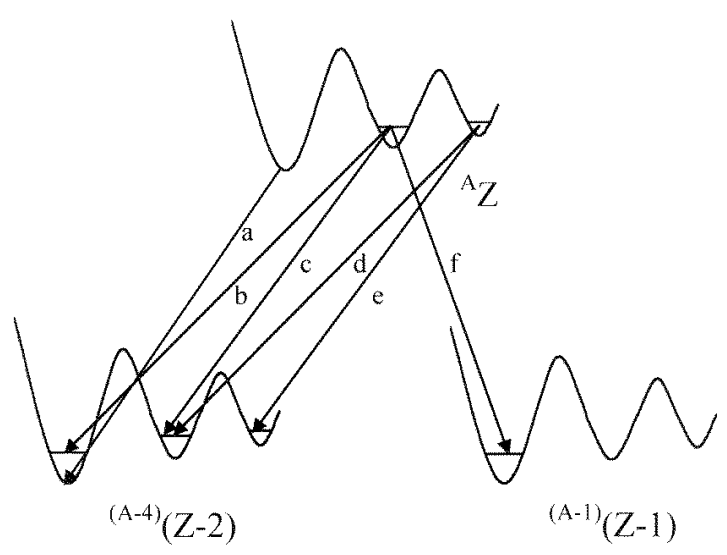

\author{
a) $\mathrm{I}^{\min } \rightarrow \mathrm{I}^{\min }$. Normal $\alpha$ 's. \\ b) $\mathrm{II}^{\text {min }} \rightarrow \mathrm{I}^{\text {min }}$. Retarded $\alpha^{\prime}$ s: \\ ${ }^{190} \mathrm{Ir} \rightarrow{ }^{186} \operatorname{Re}[28]$. \\ c) $\mathrm{II}^{\mathrm{min}} \rightarrow \mathrm{II}^{\mathrm{min}}$. Enhanced $\alpha$ 's: \\ ${ }^{210} \mathrm{Fr} \rightarrow{ }^{206} \mathrm{At} 26$. \\ $\sim{ }^{238} \mathrm{Am} \rightarrow{ }^{234} \mathrm{~Np}$ [24]. \\ d) $\mathrm{III}^{\text {min }} \rightarrow \mathrm{II}^{\text {min }}$. Retarded $\alpha$ 's: \\ ${ }^{195} \mathrm{Hg} \rightarrow{ }^{191} \mathrm{Pt}[28]$. \\ e) $\mathrm{III}^{\text {min }} \rightarrow \mathrm{III}^{\text {min }}$. Enhanced $\alpha$ 's:

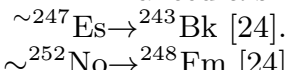 \\ f) $\mathrm{II}^{\mathrm{min}} \rightarrow \mathrm{I}^{\mathrm{min}}$. Retarded protons [27, 28$]$. \\ ${ }^{198} \mathrm{Tl} \rightarrow{ }^{197} \mathrm{Hg}(?)$ 27 \\ ${ }^{205} \mathrm{Fr} \rightarrow{ }^{204} \mathrm{Rn}(?)$,
}

FIG. 7: Summary of abnormal particle decays seen in various experiments

\section{SUPER- AND HYPER-DEFORMED ISOMERIC STATES AND THE PUZZLING PHENOMENA SEEN IN NATURE}

The discovered super- and hyper-deformed long-lived isomeric states enable one to understand the previously puzzling phenomena seen in nature (See the Introduction).

The source for the Po halos [9, 10] may be such isomeric states in isotopes with $\mathrm{Z} \simeq 84$ which decayed, by $\beta$ - or $\gamma$-decays, to the ground states of ${ }^{210} \mathrm{Po},{ }^{214} \mathrm{Po}$ and ${ }^{218} \mathrm{Po}$.

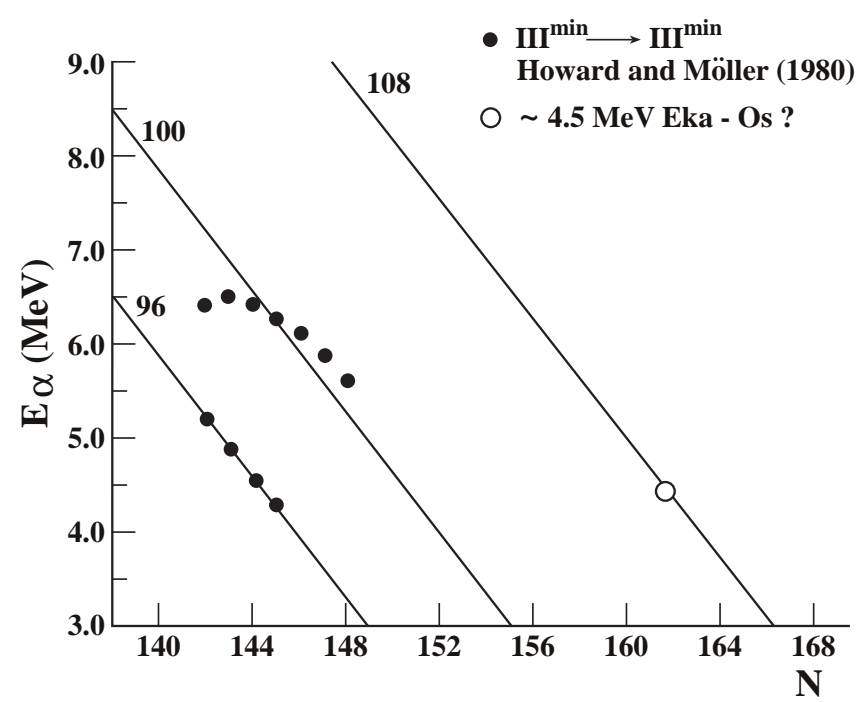

FIG. 8: Predictions [36], and extrapolations from these predictions, of the $\mathrm{III}^{\text {min }} \rightarrow \mathrm{III}^{\text {min }} \alpha$-particle energies. The black dots are the predictions for various isotopes of $\mathrm{Z}=96$ and $Z=100$. The straight lines are extrapolations from these predictions. The open circle shows the position of $4.5 \mathrm{MeV}$ $\alpha$-particles in $\mathrm{Z}=108$.
The low energy $\alpha$-particles around $4.5 \mathrm{MeV}$ 12, 13, 14, 15] can consistently be interpreted as due to a very enhanced $\mathrm{III}^{\text {min }} \rightarrow \mathrm{III}^{\text {min }}$ transition in $\mathrm{Z} \sim 108$ and $\mathrm{A} \sim 271$. The predicted [24, 26] half-life in this case is around $10^{9} \mathrm{y}$, as seen in Table V. This resolves the first difficulty in understanding these data, namely the about eight orders of magnitude shorter lifetime than what is predicted [22, 23] from energy versus lifetime relationship for a normal $\alpha$ transition. (About $2.5 \times 10^{8}$ y estimated experimentally as compared to $5 \times 10^{16} \mathrm{y}$. See the Introduction).

TABLE V: Calculated half-lives for hyperdeformed to hyperdeformed $\alpha$-particle transition of $4.5 \mathrm{MeV}$ from ${ }^{271} \mathrm{Hs}$ assuming various deformation parameters 24 .

\begin{tabular}{llll}
\hline$\beta_{2}$ & $\beta_{3}$ & $\beta_{4}$ & $\mathrm{t}_{1 / 2}(\mathrm{y})$ \\
\hline $1.2^{a}$ & $0.0^{b}$ & $0.0^{a}$ & $1.8 \times 10^{11}$ \\
$1.2^{a}$ & $0.19^{c}$ & 0.0 & $4.6 \times 10^{9}$ \\
$0.85^{d}$ & $0.35^{d}$ & $0.18^{d}$ & $1.3 \times 10^{8}$ \\
\hline
\end{tabular}

${ }^{a} \epsilon_{2}$ and $\epsilon_{4}$ values for ${ }^{248} \mathrm{Fm}$ were taken from Ref. [36] and converted to $\beta_{2}$ and $\beta_{4}$ values according to Ref. [40.

${ }^{b}$ Assuming $\beta_{2}=0$.

${ }^{c}$ Assuming $\beta_{3}=\epsilon_{3}$ of Ref. $\sqrt{36}$.

${ }^{d}$ Parameters given in Ref. 38 for ${ }^{232} \mathrm{Th}$.

In addition, an extrapolation of the deduced $\alpha$-energies for $\mathrm{III}^{\text {min }} \rightarrow \mathrm{III}^{\text {min }}$ transitions from the predictions of Ref. [36] shows that for $\mathrm{Z}=108, \mathrm{E}_{\alpha}$ of about $4.5 \mathrm{MeV}$ corresponds to $\mathrm{N} \sim 162$ (see Fig. 8). This is consistent with the suggestion [15] that ${ }^{247} \mathrm{Cm}$ may be a descendent of the superheavy element with $\mathrm{Z}=108$ which decays by the $4.5 \mathrm{MeV} \alpha$-particles, since ${ }^{247} \mathrm{Cm}$ can be obtained from ${ }_{108}^{271} \mathrm{Hs}_{163}$ by successive six $\alpha$-decays. Another possibility is that the long-lived isotope is ${ }^{267} \mathrm{Hs}$ which decays by two $\beta^{+}$or electron capture decays to ${ }^{267} \mathrm{Sg}$ which then follows by five successive $\alpha$-decays to ${ }^{247} \mathrm{Cm}$. It should however be mentioned that in principle the above 4.5 
$\mathrm{MeV} \alpha$-particles may also be due to a strongly retarded $\mathrm{II}^{\text {min }} \rightarrow \mathrm{I}^{\text {min }}$ or $\mathrm{III}^{\text {min }} \rightarrow \mathrm{II}^{\text {min }}$ transition in the region of Os itself. (For normal 4.5 MeV $\alpha$-particles in Os the expected [22, 23] half-life is about 1 y. Such short-lived nuclide can not exist in nature).

\section{SUMMARY}

It was shown that the newly discovered long-lived super- and hyper-deformed isomeric states can provide consistent interpretations to two previously unexplained phenomena seen in nature. Thus, the Po halos can be understood as due to the existence of such isomeric states in nuclei with $\mathrm{Z}$ values around 84 and atomic masses in the region of $210-218$. The observed $4.5 \mathrm{MeV} \alpha$ particle group can be understood as due to a low energy and strongly enhanced hyperdeformed to hyperdeformed transition in a nucleus with $\mathrm{Z}=108$ and $\mathrm{A} \simeq 271$.

It seems to us that the existence of superheavy elements in nature is not impossible.

\section{ACKNOWLEDGEMENTS}

We appreciate very much the valuable discussions with J. L. Weil and N. Zeldes.
[1] V. M. Strutinskii, Yadernaya Fizika 3, 614 (1964).

[2] W. D. Myers and W. J. Swiateski, Nucl. Phys. 81, 1 (1966).

[3] A. Sobiczewski, F. A. Gareev and B. N. Kalinkin, Phys. Lett. 22, 590 (1966).

[4] V. M. Strutinskii, Nucl. Phys. A95, 420 (1967).

[5] C. L. Wong, Phys. Rev. Lett. 19, 328 (1967).

[6] Yu. A. Muzychka, V. V. Pashkevich and Strutinskii, Dubna Preprint R7-3733, 1968.

[7] S. G. Nilsson, J. R. Nix, A. Sobiczewski, Z. Szymanski, S. Wycech, C. Gustafson and P. Möller, Nucl. Phys. A115, 545 (1968).

[8] J. Grumann, U. Mosel, B. Fink and W. Greiner, Z. Physik 228, 371 (1969).

[9] G. H. Henderson and F. W. Sparks, Proc. Roy. Soc. Lond., A173, 238 (1939).

[10] R. V. Gentry, Science 160, 1228 (1968).

[11] R. V. Gentry, Creation's Tiny Mystery, Earth Science Associates, Knoxville, Tennessee (1992).

[12] V. V. Cherdyntsev and V. F. Mikhailov, Geochemistry No. 1, 1 (1963).

[13] R. D. Chery, K. A. Richardson and J. A. S. Adams, Nature 202, 639 (1964).

[14] V. V. Cherdyntsev, V. L. Zverev, V. M. Kuptsov and G. I. Kislitsina, Geochemistry No. 4, 355 (1968).

[15] H. Meier et al., Z. Naturforsch. 25, 79 (1970).

[16] J. Joly, J. Phil. Mag. 13, 381 (1907).

[17] O. Mugge, Zent. Mineral. 1907, 397 (1907).

[18] N. Feather, Cumm. Roy. Soc. Edinburgh, No. 11, 147 (1978).

[19] P. Möller, J. R. Nix and K. -L. Kratz, At. Data Nucl. Data Tables 66, 131 (1997).

[20] H. Koura, M.Uno, T. Tachibana and M.Yamada, Nucl. Phys. A674, 47 (2000); RIKEN-AF-NP-394 (April 2001).

[21] S. Liran, A. Marinov and N. Zeldes, Phys. Rev. C62, 047301 (2000); arXiv:nucl-th/0102055.

[22] V. E. Viola, Jr. and G. T. Seaborg, J. Inorg. Nucl. Chem. 28, 741 (1966).

[23] G. Royer, J. Phys. G: Nucl. Part. Phys. 26, 1149 (2000).

[24] A. Marinov, S. Gelberg, D. Kolb and J. L. Weil, Int. J. Mod. Phys. E10, 209 (2001).

[25] A. Marinov, C. J. Batty, A. I. Kilvington, G. W. A. Newton, V. J. Robinson and J. D. Hemingway, Nature 229,
464 (1971).

[26] A. Marinov, S. Gelberg and D. Kolb, Mod. Phys. Lett. A11, 861 (1996).

[27] A. Marinov, S. Gelberg and D. Kolb, Mod. Phys. Lett. A11, 949 (1996).

[28] A. Marinov, S. Gelberg and D. Kolb, Int. J. Mod. Phys. E10, 185 (2001).

[29] A. Marinov, S. Gelberg, D. Kolb, R. Brandt and A. Pape, To be published in the Proc. 3rd Int. Conf. on Exotic Nuclei and Atomic Masses, July 2-7, 2001, Hämeenlinna, Finland.

[30] A Marinov, S. Eshhar and D. Kolb, Phys. Lett. B191, 36 (1987).

[31] W. Satula, S. Ćwiok, W. Nazarewicz, R. Wyss and A. Johnson, Nucl. Phys. A529, 289 (1991).

[32] S. J. Krieger, P. Bonche, M. S. Weiss, J. Meyer, H. Flocard and P. -H. Heenen, Nucl. Phys. A542, 43 (1992).

[33] G. Audi and A. H. Wapstra, Nucl. Phys. A565, 66 (1993).

[34] J. Fernández-Neillo, C. H. Dasso and S. Landowne, Code CCDEF, Comp. Phys. Comm. 54, 409 (1985).

[35] S. Raman, C. H. Malarkey, W. T. Milner, C. W. Nestor, Jr. and P. H. Stelson, At. Data and Nucl. Data Tables 36, 1 (1987).

[36] W. M. Howard and P. Möller, At. Data and Nucl. Data Tables 25, 219 (1980).

[37] W. Nazarewicz, Phys. Lett. B305, 195 (1993).

[38] S. Ćwiok, W. Nazarewicz, J. X. Saladin, W. Plóciennik and A. Johnson, Phys. Lett. B322, 304 (1994).

[39] A. Krasnahorkay et al., Phys. Rev. Lett. 80, 2073 (1998).

[40] W. Nazarewicz and I. Ragnarsson, Handbook of Nuclear Properties, eds. D. N. Poenaru and W. Greiner (Clarendon Press, Oxford, 1996) p. 80.

[41] S. Hofmann et al., Z. Phys. A354, 229 (1996).

[42] A. Marinov, S. Gelberg and D. Kolb, Inst. Phys. Conf. Ser. No. 132: Section 4, Nuclei Far from Stability/Atomic Masses and Fundamental Constants 1992, p. 437.

[43] R. B. Firestone, V. S. Shirley, C. M. Baglin, S. Y. F. Chu and J. Zipkin, Table of Isotopes (Wiley-Interscience, 1996).

[44] S. G. Nilson, G. Ohlén, C. Gustafson and P. Möller, Phys. Lett. 30B, 437 (1969). 